\title{
Nannomecoptera and Neomecoptera - an overview
}

Nannochoristidae (=Nannomecoptera) and Boreidae (=Neomecoptera) are traditionally assigned to the holometabolan order Mecoptera. Both are presently in the focus of insect systematists. They differ strikingly from the "typical" mecopteran pattern, not only in their larval and adult morphology but also in their lifestyle and reproductive biology. Even recent analyses of extensive molecular data sets in the 1KITE project (www.1kite.org) could not clarify the phylogenetic affinities of the two taxa. Both of them display fascinating features, such as preferred temperatures of around $0^{\circ} \mathrm{C}$ in the case of Boreidae and extremely slender larvae adapted to running water in the case of Nannochoristidae. Despite the very low number of known species, there is no doubt that both groups are key taxa and in their own way highlights in the evolution of the extremely successful Holometabola. This and an impressive number of recent studies on various aspects of Nannochoristidae and Boreidae more than justify a new volume in the Handbook of Zoology series (De Gruyter) dedicated to these highly unusual taxa. 
